\title{
Martelli Chaotic Properties of a Generalized Form of Zadeh's Extension Principle
}

\author{
Yaoyao Lan ${ }^{1,2}$ and Chunlai $\mathrm{Mu}^{3}$ \\ ${ }^{1}$ College of Computer Science, Chongqing University, Chongqing 401331, China \\ ${ }^{2}$ Key Laboratory of Data Analyzing and Image Processing, Department of Mathematics and Finance, \\ Chongqing University of Arts and Sciences, Yongchuan 402160, China \\ ${ }^{3}$ College of Mathematics and Statistics, Chongqing University, Chongqing 401331, China \\ Correspondence should be addressed to Yaoyao Lan; yylan81@hotmail.com
}

Received 9 October 2013; Revised 6 February 2014; Accepted 25 February 2014; Published 31 March 2014

Academic Editor: Marcelo A. Savi

Copyright (C) 2014 Y. Lan and C. Mu. This is an open access article distributed under the Creative Commons Attribution License, which permits unrestricted use, distribution, and reproduction in any medium, provided the original work is properly cited.

Let $X$ denote a compact metric space and let $f: X \rightarrow X$ be a continuous map. It is known that a discrete dynamical system $(X, f)$ naturally induces its fuzzified counterpart, that is, a discrete dynamical system on the space of fuzzy compact subsets of $X$. In 2011 , a new generalized form of Zadeh's extension principle, so-called $g$-fuzzification, had been introduced by Kupka 2011. In this paper, we study the relations between Martelli's chaotic properties of the original and $g$-fuzzified system. More specifically, we study the transitivity, sensitivity, and stability of the orbits in system $(X, f)$ and its connections with the same ones in its $g$-fuzzified system.

\section{Introduction}

The main goal of the theory of discrete dynamical system is to understand the asymptotic properties and topological structures of the orbits. In certain sense, the study of the orbits in discrete dynamical system is to investigate the movement of the points in the base space. In many cases, however, it is not sufficient to know how the points move, but it is necessary to understand the motion of the subsets of base space (e.g., in migration phenomenon), and this leads us to the problem of analyzing the dynamics of the set-valued discrete dynamical systems. In this direction, many elegant results have been obtained (please see [1-7] and the references cited therein).

As the complexity of research subjects increased, an accurate description for systems becomes more and more difficult, and the situation would become more complicated when the systems are affected by the uncertainty. In this case, the fuzzy system should be considered. It is well known that any given discrete dynamical system uniquely induces its fuzzified counterpart, that is, a discrete system on the space of fuzzy sets. It is natural to ask the following question: what is the relation between dynamical properties of the original and fuzzified systems?
Motivated by this question, the study of discrete fuzzy dynamical systems has recently become active [8-12]. As a partial response to the question above, in the case of Devaney chaos [13], the transitivity, periodic density, and sensitivity between two systems have been analyzed in 2008 [14]. In addition, by analyzing connections between the fuzzified dynamical systems related to the original one, the authors have pointed out that this kind of investigation should be useful in many real problems, such as in ecological modelling and demographic sciences. Some recent works along these lines appear. In 2011, Kupka proves that there exists a transitive fuzzification on the space of normal fuzzy sets, which contains the solution of the problem that was partially solved in [14]. Specifically, the author considers a symbolic dynamical system as the original system and then shows that Zadeh's extension of the shift map is transitive. As regards the periodic density, a concept of piecewise constant fuzzy set is introduced, and then period density equivalence of $f$ and $\widehat{f}$ is proposed. Consequently, the question has been completely solved [15]. And then we discuss this issue by using the weakly mixing property [16].

Among the methods of fuzzification, Zadeh's extension [17] is often used, but it can lose information that is carried 
by the original system. Therefore, more general extension principles have been developed $[18,19]$. Recently, a concept of $g$-fuzzification, which allows us to modify the membership grades of points in each iteration, has been introduced [19]. The use of $g$-fuzzification is quite natural but differs slightly from Zadeh's extension principle, and it can be useful in several situations. Take a fuzzy set "old people" for example; old people in ancient time are not considered as old at present since the average age of people is increasing. We also can find several examples to illustrate such kinds of fuzzy sets with variable membership grades. Zadeh's extension, however, does not reflect this fact. On the other hand, the situation becomes more complicated in fuzzy control. In [20], the authors show that a chaotic function on $\mathbb{R}^{n}$, for its fuzzification in the sense of Zadeh, is degenerate, because the iterates are asymptotically crisp and, ultimately, we obtain chaos of a mapping of ordinary sets rather than of fuzzy output. In this case, the usual fuzzification is inadequate to describe complexities which may arise in fuzzy control. Consequently, a concept of $\Gamma$-fuzzification has been developed in [18], which does not degenerate under chaotic iteration. Now, as a new generalized extension principle, $g$-fuzzification includes the usual fuzzification (Zadeh's extension) and $\Gamma$-fuzzification as two special cases of it, and the developed methods enable us to study the dynamics of discrete fuzzy systems in a more efficient way.

Chaotic dynamics has been hailed as the third great scientific revolution of the 20th century, along with relativity and quantum mechanics. But there is not a generally accepted definition of chaos yet. The different definitions of chaos being around have been designed to meet different purposes and they are based on very different backgrounds and levels of mathematical sophistication. To compare various kinds of definitions of chaos naturally attracts the interest of many researchers. In 2002, Huang and Ye showed that chaos in the sense of Devaney is stronger than that of Li-Yorke [21]. The conclusion stimulates the study of the relations between different definitions of chaos [22-24].

Among various definitions of chaos, Martelli's chaos is one of the definitions of chaos which are suitable for easy and reliable numerical verification [25]. The authors make comparison of different definitions of chaos and point out that Martelli's chaos embodies the essential features which all other definitions are trying to capture [26]. It is worth noting that although formulated in a different way, Martelli's chaos is practically equivalent to chaos in the sense of Wiggins [27]. There remains, however, a difference between the two definitions. Wiggins does not require sensitivity with respect to the base space, while Martelli requires instability with respect to the base space.

In this paper, we focus on relations between Martelli's chaotic properties of the original and $g$-fuzzified dynamical systems. Below, Section 2 gives basic notions and definitions. Section 3 discusses the relation between Martelli's chaotic properties of the original and $g$-fuzzified systems. A brief conclusion concludes the paper.

\section{Preliminaries}

In this section, we complete notations and recall some known definitions. Let $f: X \rightarrow X$ be a continuous map acting on a compact metric space $(X, d)$. An orbit of a point $x_{0} \in X$ is the set $\left\{f^{n}\left(x_{0}\right): n \geq 0\right\}$, denoted by orb $\left(x_{0}, f\right)$ or simply $\operatorname{orb}\left(x_{0}\right)$ when the function $f$ is clearly specified. A point $y$ is a limit point of orb $\left(x_{0}\right)$ if a subsequence of orb $\left(x_{0}\right)$ converges to $y$. The set of limit points of orb $\left(x_{0}\right)$ is denoted by $L\left(x_{0}\right)$.

We say that $f$ is transitive if for any pair of nonempty open sets $U$ and $V$ there exists $n \geq 1$ such that $f^{n}(U) \cap V \neq \emptyset ; f$ is point transitive if there exists a point $x_{0} \in X$ such that the orbit of $x_{0}$ is dense in $X$; that is, $\overline{\operatorname{orb}\left(x_{0}\right)}=X$, and $x_{0}$ is called a transitive point of $X$.

We say that $\operatorname{orb}(x)$ is unstable if there exists $\delta_{x}$ such that, for any neighborhood $U$ of $x$, there exist $y \in U$ and $n \geq 0$ such that $d\left(f^{n}(x), f^{n}(y)\right)>\delta_{x}$. An orbit which is not unstable is said to be stable.

We say that $f$ has sensitive dependence on initial conditions if there is a constant $\delta>0$ such that for every point $x$ and every neighborhood $U$ about $x$ there are a $y \in U$ and a $k \geq 1$ such that $d\left(f^{k}(x), f^{k}(y)\right) \geq \delta$. Hence, every orbit orb $(x)$ with $x \in X$ is unstable with the same constant $\delta$. Consequently, sensitive dependence on initial conditions is stronger than instability.

Definition 1 (see [25]). Let $(X, d)$ be a compact metric space and let $f: X \rightarrow X$ be continuous. Then, $f$ is Martelli chaotic provided that there exists $x_{0} \in X$ such that

(i) $L\left(x_{0}\right)=X$;

(ii) $\operatorname{orb}\left(x_{0}\right)$ is unstable.

In this research, we call a Martelli chaotic map M-chaotic for short.

Below, we present some definitions from fuzzy theory. Let $\mathscr{K}(X)$ be the class of all nonempty and compact subsets of $X$. If $A \in \mathscr{K}(X)$, we define the $\varepsilon$-neighbourhood of $A$ as the set

$$
N(A, \varepsilon)=\{x \in X \mid d(x, A)<\varepsilon\},
$$

where $d(x, A)=\inf _{a \in A}\|x-a\|$.

The Hausdorff separation $\rho(A, B)$ of $A, B \in \mathscr{K}(X)$ is defined by

$$
\rho(A, B)=\inf \{\varepsilon>0 \mid A \subseteq N(B, \varepsilon)\} .
$$

The Hausdorff metric on $\mathscr{K}(X)$ is defined by letting

$$
H(A, B)=\max \{\rho(A, B), \rho(B, A)\} .
$$

Define $\mathscr{F}(X)$ as the class of all upper semicontinuous fuzzy sets $u: X \rightarrow[0,1]$ such that $[u]_{\alpha} \in \mathscr{K}(X)$, where $\alpha$ cuts and the support of $u$ are defined by

$$
\begin{aligned}
{[u]_{\alpha} } & =\{x \in X \mid u(x) \geq \alpha\}, \quad \alpha \in[0,1], \\
\operatorname{supp}(u) & =\overline{\{x \in X \mid u(x)>0\},}
\end{aligned}
$$

respectively. 
Moreover, let $\mathscr{F}_{0}(X)$ denote the space of all nonempty fuzzy sets on $X$ and let $\emptyset_{X}$ denote the empty fuzzy set $\left(\emptyset_{X}(x)=\right.$ 0 for all $x \in X)$.

A level-wise metric $d_{\infty}$ on $\mathscr{F}(X)$ is defined by

$$
d_{\infty}(u, v)=\sup _{\alpha \in[0,1]} H\left([u]_{\alpha},[v]_{\alpha}\right)
$$

for all $u, v \in \mathscr{F}(X)$. It is well known that if $(X, d)$ is complete, then $\left(\mathscr{F}(X), d_{\infty}\right)$ is also complete but is not compact and is not separable (see $[19,28,29])$.

Lemma 2 (see $[9,14])$. Let $A$ be an open subset of $X$. Define $e(A)=\left\{u \in \mathscr{F}(X):[u]_{0} \subseteq A\right\}$, and then $e(A)$ is an open subset of $\mathscr{F}(X)$.

Let $f: X \rightarrow X$ be continuous. A usual fuzzification (often called Zadeh's extension) $\widehat{f}: \mathbb{F}(X) \rightarrow \mathbb{F}(X)$ is defined by

$$
\widehat{f}(u)(x)=\sup _{y \in f^{-1}(x)} u(y)
$$

for any $u \in \mathbb{F}(X)$ and $x \in X$.

Now let us introduce $g$-fuzzification. Denote $D_{m}(I)$ as the set of all nondecreasing right continuous functions $g: I \rightarrow I$ for which $g(x)=x$ if $x=0$ and $x=1$. Let $C_{m}(I)$ be the set of all continuous maps from $D_{m}(I)$. For any $g \in D_{m}(I)$, a $g$-fuzzification $\widehat{f}_{g}: \mathbb{F}(X) \rightarrow \mathbb{F}(X)$ is defined by

$$
\widehat{f}_{g}(u)(x)=\sup _{y \in f^{-1}(x)}\{g(u(y))\}
$$

for any $u \in \mathbb{F}(X), x \in X$.

An $\alpha$-cut $[u]_{\alpha}^{g}$ of a fuzzy set $u \in \mathbb{F}(X)$ with respect to $g \in$ $D_{m}(I)$ is

$$
[u]_{\alpha}^{g}=\{x \in \operatorname{supp}(u) \mid g(u(x)) \geq \alpha\} \quad \text { for } \alpha \in(0,1] .
$$

Lemma 3 (see [19]). Let $f: X \rightarrow X$ be continuous and let $\widehat{f}_{g}$ be $g$-fuzzification. Then,

$$
f\left([u]_{\alpha}^{g}\right)=\left[\widehat{f}_{g}(u)\right]_{\alpha}
$$

holds for any $u \in \mathbb{F}_{0}(X), g \in D_{m}(I)$, and $\alpha \in(0,1]$.

Lemma 4 (see [19]). Let $g \in D_{m}(I), u \in \mathbb{F}_{0}(X)$, and $\alpha \in(0,1]$. If $[u]_{\alpha}^{g} \neq \infty$. then there is $c \in(0,1]$ such that $[u]_{\alpha}^{g}=[u]_{c}$.

\section{M-Chaotic Relations between $\widehat{f}_{g}$ and $f$}

In this section, we study the relations between Martelli's chaotic properties of the original system $(X, f)$ and $g$-fuzzified system $\left(\mathbb{F}(X), \widehat{f}_{g}\right)$, where $\mathbb{F}(X)$ is equipped with the level-wise topology, that is, the metric topology induced by $d_{\infty}$.

On the one hand, some conditions are discussed, under which $\widehat{f}_{g} M$-chaotic implies $f M$-chaotic. On the other hand, several examples are presented to illustrate that, in general, $f M$-chaotic does not imply $\widehat{f}_{g} M$-chaotic.
Proposition 5. Define $[u]_{0}^{g}=\left\{x \in[u]_{0} \mid g(u(x))>0\right\}$. Then

$$
[u]_{0}^{g}=[u]_{0}
$$

holds for every $u \in \mathbb{F}_{0}(X)$ and $g \in D_{m}(I)$.

Proof. The inclusion $[u]_{0}^{g} \subseteq[u]_{0}$ follows directly from the definition of $[u]_{0}^{g}$. If $x \in[u]_{0}$, then, because $g$ is nondecreasing, we have $g(u(x))>0$, which implies $x \in[u]_{0}^{g}$, and, consequently, $[u]_{0}^{g} \supseteq[u]_{0}$ holds.

Proposition 6. Let $U$ be subset of $X$ and let $f: X \rightarrow X$ be continuous. Then, $\widehat{f}_{g}(e(U)) \subseteq e(f(U))$.

Proof. If $u \in \widehat{f}_{g}(e(U))$, then there exists $u^{*} \in e(U)$ such that $u=\widehat{f}_{g}\left(u^{*}\right)$. Hence, due to Lemma 3 and Proposition 5, we have that $[u]_{0}=\left[\widehat{f}_{g}\left(u^{*}\right)\right]_{0}=f\left(\left[u^{*}\right]_{0}^{g}\right)=f\left(\left[u^{*}\right]_{0}\right)$, since $\left[u^{*}\right]_{0} \subseteq U$ and $[u]_{0}=f\left(\left[u^{*}\right]_{0}\right) \subseteq f(U)$; thus $u \in e(f(U))$, and the inclusion follows.

Proposition 7. Let $u \in \mathbb{F}_{0}(X), g \in D_{m}(I)$, and $\alpha_{i} \in(0,1]$, $i=1, \ldots, n, \ldots$. Then, there exists an $\alpha_{n} \in(0,1]$ such that $\left[\widehat{f}_{g}^{n}(u)\right]_{\alpha_{n}}=\left[\widehat{f_{g}^{n}}(u)\right]_{\alpha_{2 n-1}}=f^{n}\left([u]_{\alpha_{2 n}}\right)$.

Proof. We do the proof by mathematical induction.

When $n=1$, by Lemmas 3 and 4 , the formula gives us $\left[\widehat{f}_{g}(u)\right]_{\alpha_{1}}=f\left([u]_{\alpha_{1}}^{g}\right)=f\left([u]_{\alpha_{2}}\right)$; therefore, the statement holds for $n=1$.

Assume that the statement is true for $n=k$; that is,

$$
\left[\hat{f}_{g}^{k}(u)\right]_{\alpha_{k}}=\left[\widehat{f_{g}^{k}}(u)\right]_{\alpha_{2 k-1}}=f^{k}\left([u]_{\alpha_{2 k}}\right) \text {. }
$$

Note that $\left[\widehat{f}_{g}^{k}(u)\right]_{\alpha_{k+2}}=f^{k}\left([u]_{\alpha_{2 k+2}}\right)$.

When $n=k+1$,

$$
\begin{aligned}
{\left[\hat{f}_{g}^{k+1}(u)\right]_{\alpha_{k+1}} } & =\left[\hat{f}_{g} \hat{f}_{g}^{k}(u)\right]_{\alpha_{k+1}} \\
& =f\left(\left[\hat{f}_{g}^{k}(u)\right]_{\alpha_{k+1}}^{g}\right)=f\left(\left[\hat{f}_{g}^{k}(u)\right]_{\alpha_{k+2}}\right) \\
& =f\left(f^{k}\left([u]_{\alpha_{2 k+2}}\right)\right)=f^{k+1}\left([u]_{\alpha_{2(k+1)}}\right) .
\end{aligned}
$$

On the other hand, $f^{k+1}\left([u]_{\alpha_{2(k+1)}}\right)=f^{k+1}\left([u]_{\alpha_{2(k+1)-1}}^{g}\right)=$ $\left[\widehat{f_{g}^{k+1}}(u)\right]_{\alpha_{2 k+1}}$.

This completes the proof.

Remark 8. The proof of Proposition 7 can also be done as follows.

Due to Lemmas 3 and 4, we have that

$$
\begin{aligned}
{\left[\widehat{f}_{g}^{n}(u)\right]_{\alpha_{n}} } & =\left[\widehat{f}_{g} \widehat{f}_{g}^{n-1}(u)\right]_{\alpha_{n}} \\
& =f\left(\left[\hat{f}_{g}^{n-1}(u)\right]_{\alpha_{n}}^{g}\right)=f\left(\left[\hat{f}_{g}^{n-1}(u)\right]_{\alpha_{n+1}}\right) \\
& =f\left(\left[\widehat{f}_{g} \widehat{f}_{g}^{n-2}(u)\right]_{\alpha_{n+1}}\right)=f^{2}\left(\left[\hat{f}_{g}^{n-2}(u)\right]_{\alpha_{n+1}}^{g}\right)
\end{aligned}
$$




$$
\begin{aligned}
& =f^{2}\left(\left[\widehat{f}_{g}^{n-2}(u)\right]_{\alpha_{n+2}}\right) \\
& \vdots \\
& =f^{n}\left([u]_{\alpha_{2 n-1}}^{g}\right)=f^{n}\left([u]_{\alpha_{2 n}}\right) .
\end{aligned}
$$

On the other hand, by Lemma 3 again, we obtain $f^{n}\left([u]_{\alpha_{2 n-1}}^{g}\right)=\left[\widehat{f_{g}^{n}}(u)\right]_{\alpha_{2 n-1}}$, and, consequently, $\left[\widehat{f}_{g}^{n}(u)\right]_{\alpha_{n}}=$ $\left[\widehat{f_{g}^{n}}(u)\right]_{\alpha_{2 n-1}}=f^{n}\left([u]_{\alpha_{2 n}}\right)$ holds.

Theorem 9. Let $u_{0}$ be a transitive point of $\left(\mathbb{F}(X), \widehat{f}_{g}\right)$. Then, every $x \in\left[u_{0}\right]_{\alpha}^{g}$ is a transitive point of $(X, f)$ for $\alpha \in(0,1]$.

Proof. Since $u_{0}$ is a transitive point of $\left(\mathbb{F}(X), \widehat{f}_{g}\right)$, there exists $k \in \mathbb{N}$ such that $d_{\infty}\left(\hat{f}_{g}^{k}\left(u_{0}\right), v\right)<\varepsilon$ for any $v \in \mathbb{F}(X)$ and $\varepsilon>0$. By using Proposition 7 and Lemma 3, we obtain

$$
\begin{aligned}
d_{\infty}\left(\widehat{f}_{g}^{k}\left(u_{0}\right), v\right) & =\sup _{\beta \in[0,1]} H\left(\left[\widehat{f}_{g}^{k}\left(u_{0}\right)\right]_{\beta},[\nu]_{\beta}\right) \\
& =\sup _{\alpha, \beta \in(0,1]} H\left(\left[\widehat{f_{g}^{k}}\left(u_{0}\right)\right]_{\alpha},[\nu]_{\beta}\right) \\
& =\sup _{\alpha, \beta \in(0,1]} H\left(f^{k}\left[u_{0}\right]_{\alpha}^{g},[\nu]_{\beta}\right)<\varepsilon,
\end{aligned}
$$

for some $\alpha, \beta \in[0,1]$. Hence, for each $y \in[\nu]_{\beta}$, there exists $x \in\left[u_{0}\right]_{\alpha}^{g}$ such that $d(x, y)<\varepsilon$, which means that every $x \in$ $\left[u_{0}\right]_{\alpha}^{g}$ is a transitive point of $(X, f)$.

Theorem 10. If $L\left(u_{0}\right)=\mathbb{F}(X)$, then there exists $x_{0} \in\left[u_{0}\right]_{\alpha}^{g}$ such that $L\left(x_{0}\right)=X$.

Proof. It follows directly from Theorem 9.

The following example shows that, in general, the converse of Theorem 10 is not true.

Example 11 (irrational rotation of circle). Let $\lambda$ be an irrational number and $R_{\lambda}: S^{1} \rightarrow S^{1}$ is defined by $R_{\lambda}\left(e^{i \theta}\right)=$ $e^{i(\theta+2 \pi \lambda)}$. It is well known that, for each $z \in S^{1}$, the orbit of $z$ is dense in $S^{1}$ and, consequently, $L(z)=S^{1}$. Nevertheless, it is not necessary for some $v \in \mathbb{F}\left(S^{1}\right)$ to exist such that $L(v)=$ $\mathbb{F}\left(S^{1}\right)$. In fact, assume that $u \in \mathbb{F}\left(S^{1}\right)$ and $\operatorname{diam}\left([u]_{0}^{g}\right)=1$. Given that $0<\varepsilon<1 / 2$, let $U=B(\widehat{1}, \varepsilon / 2)$ and $V=B(u, \varepsilon / 2)$, and by Proposition 5, we obtain

$$
\begin{gathered}
\omega \in U=B\left(\widehat{1}, \frac{\varepsilon}{2}\right) \Longrightarrow \operatorname{diam}\left([\omega]_{0}^{g}\right)=\operatorname{diam}\left([\omega]_{0}\right) \leq \frac{\varepsilon}{2}, \\
\nu \in V=B\left(u, \frac{\varepsilon}{2}\right) \Longrightarrow \operatorname{diam}\left([\nu]_{0}^{g}\right)=\operatorname{diam}\left([\nu]_{0}\right) \geq 1-\varepsilon,
\end{gathered}
$$

since

$$
\operatorname{diam}\left(\left[\widehat{R}_{\lambda_{g}}^{n}(v)\right]_{0}\right)=\operatorname{diam}\left(R_{\lambda}^{n}[v]_{0}\right) \geq 1-\varepsilon
$$

for $n \in \mathbb{N}$. Hence, $U \cap \widehat{R}_{\lambda_{g}}^{n}(V)=\emptyset$, which means that there exists no $\nu \in V$ such that $\widehat{R}_{\lambda_{g}}^{n}(\nu)=\omega$ for some $\omega \in \mathbb{F}(X)$, and, consequently, $L(\nu) \neq \mathbb{F}(X)$.
Theorem 12. Let $f: X \rightarrow X$ be continuous, let $\widehat{f}_{g}$ be the $g$ extension of $f$, and let $u_{0} \in \mathbb{F}(X)$. If the orbit of $u_{0}$ is unstable in $\mathbb{F}(X)$, then there exists $x_{0} \in\left[u_{0}\right]_{\beta}^{g}$ such that the orbit of $x_{0}$ is unstable in $X$, where $\beta \in[0,1]$.

Proof. Let the assumptions be satisfied. Then, there exists $\delta_{u_{0}}$ such that for every $\epsilon>0$ we can find $\nu \in \mathbb{F}(X)$ and $k \in \mathbb{N}$ satisfying $\nu \in B\left(u_{0}, \varepsilon\right)$ and

$$
\begin{aligned}
d_{\infty} & \left(\widehat{f}_{g}^{k}\left(u_{0}\right), \hat{f}_{g}^{k}(\nu)\right) \\
= & \sup _{\alpha \in[0,1]} H\left(\left[\hat{f}_{g}^{k}\left(u_{0}\right)\right]_{\alpha},\left[\hat{f}_{g}^{k}(\nu)\right]_{\alpha}\right) \\
= & \sup _{\beta, \gamma \in[0,1]} H\left(\left[\widehat{f}_{g}^{k}\left(u_{0}\right)\right]_{\beta},\left[\widehat{f_{g}^{k}}(\nu)\right]_{\gamma}\right) \\
= & \sup _{\beta, \gamma \in[0,1]} H\left(f^{k}\left[u_{0}\right]_{\beta}^{g}, f^{k}[\nu]_{\gamma}^{g}\right)>\delta_{u_{0}} .
\end{aligned}
$$

Thus, there exist $x_{0} \in\left[u_{0}\right]_{\beta}^{g}$ and $y_{0} \in[\nu]_{\gamma}^{g}$ such that $d\left(x_{0}\right.$, $\left.y_{0}\right)>\delta_{u_{0}}$. Since $\nu \in B\left(u_{0}, \varepsilon\right)$, we have $d\left(x_{0}, y_{0}\right)<\varepsilon$. This proves that there exists $x_{0} \in\left[u_{0}\right]_{\beta}^{g}$ such that the orbit of $x_{0}$ is unstable in $X$ with instable constant $\delta_{u_{0}}$.

Example 13. Consider the foregoing example (Example 11); because $R_{\lambda}$ is isometric, it does not exhibit sensitive dependence on initial conditions and hence the orbit of each $z \in S^{1}$ is stable, which implies that, by Theorem 12, there exists no orbit of $u \in \mathbb{F}\left(S^{1}\right)$ that is unstable.

By combining Theorems 9, 10, and 12, we obtain the following theorem.

\section{Theorem 14. If $\widehat{f}_{g}$ is $M$-chaotic, then $f$ is $M$-chaotic.}

We will need some notions from Denjoy map [13]. Recall that the circle $S^{1}$ can be considered as the quotient space $\mathbb{R} / \mathbb{Z}$, where $\mathbb{R}$ and $\mathbb{Z}$ are the sets of real numbers and integers, respectively. The irrational rotation of the circle $R_{\lambda}: S^{1} \rightarrow S^{1}$ is then given by

$$
R_{\lambda}(x)=x+\lambda(\bmod 1)
$$

where $\lambda$ is irrational. Recall that a Denjoy map can be constructed as follows. Take any point $x_{0} \in S^{1}$. We cut out each point $R_{\lambda}^{n}\left(x_{0}\right)$ on the orbit of $x_{0}$ and replace it with a small interval $I_{n}$. For $n \in \mathbb{N}$,

(a) $\mathscr{L}\left(I_{0}\right)=1 / 4, \mathscr{L}\left(I_{n+1}\right)<\mathscr{L}\left(I_{n}\right), \mathscr{L}\left(I_{n}\right)=\mathscr{L}\left(I_{-n}\right)$, and $\sum_{n \in \mathbb{Z}} \mathscr{L}\left(I_{n}\right)=1$, where $\mathscr{L}\left(I_{n}\right)$ denotes the length of the interval $I_{n}$;

(b) $\lim _{n \rightarrow \infty}\left(\mathscr{L}\left(I_{n+1}\right) / \mathscr{L}\left(I_{n}\right)\right)=1$.

Consequently, a new circle $S^{*}$ has been constructed. The Denjoy homeomorphism $D_{\lambda}: S^{*} \rightarrow S^{*}$ is an orientation preserving homeomorphism of $S^{*}$. There exists a Cantor set $C_{\lambda} \subset S^{*}$ on which $D_{\lambda}$ acts minimally. It is known that there exists a continuous surjection $h_{\lambda}: S^{*} \rightarrow S^{1}$ that semiconjugates $D_{\lambda}$ with $R_{\lambda}$. In [30], the authors show that the system $\left(\mathbb{K}\left(C_{\lambda}\right), D_{\lambda}\right)$ is not sensitive. 
Proposition 15. Let $x \in C_{\lambda}$; then the orbit of $x$ is unstable in $\left(C_{\lambda}, D_{\lambda}\right)$ with the constant $1 / 4$.

Proof. Suppose that $y \in B(x, \varepsilon)$ and $h_{\lambda}(x) \neq h_{\lambda}(y)$ for any $\varepsilon>0$. Since the orbit of $x_{0}$ is dense in $S^{1}$, there exist some $k \in \mathbb{N}$ such that $R_{-\lambda}^{k}\left(x_{0}\right) \in\left[h_{\lambda}(x), h_{\lambda}(y)\right]$, where $\left[h_{\lambda}(x)\right.$, $\left.h_{\lambda}(y)\right]$ is the closed $\operatorname{arc}$ in $S^{1}$. Thus, we have $x_{0} \in R_{\lambda}^{k}\left(\left[h_{\lambda}(x)\right.\right.$, $\left.\left.h_{\lambda}(y)\right]\right)$. Consequently, due to the construction of Denjoy map, we obtain $I_{0} \subset\left[D_{\lambda}^{k}(x), D_{\lambda}^{k}(y)\right]$, which means that $d\left(D_{\lambda}^{k}(x), D_{\lambda}^{k}(y)\right)>1 / 4$.

The following proposition shows that the instability of the orbit in $\left(C^{\lambda}, D_{\lambda}\right)$ cannot be inherited by its $g$-fuzzification. More specifically, there exist points arbitrarily close to $u \in$ $\mathbb{F}\left(C_{\lambda}\right)$ which eventually also close to $u$ under iteration of $\widehat{D_{\lambda_{g}}}$, although there exist some $x \in[u]_{0}$ such that the orbits of these points are unstable in $\left(C^{\lambda}, D_{\lambda}\right)$. It should be mentioned that our approach was inspired by the idea in [8] where a continuous map $i_{\lambda}$ was defined.

Define $i_{\lambda}: \mathbb{K}\left(C_{\lambda}\right) \rightarrow \mathbb{F}\left(C_{\lambda}\right)$ by $i_{\lambda}(K)=\lambda \chi_{K}$ for any $K \in \mathbb{K}\left(C_{\lambda}\right)$ and any $\lambda \in(0,1]$, where $\chi_{K}$ is the characteristic function of $K$ (that is to say, $\chi_{K}(x)=1$ if $x \in K$ and $\chi_{K}(x)=0$ if $x \notin K)$. Hence, $i_{\lambda} \circ \bar{D}_{\lambda}=\widehat{D_{\lambda g}} \circ i_{\lambda}$. Note that $i_{\lambda}$ is continuous.

Proposition 16. Let $u \in \mathbb{F}\left(C_{\lambda}\right)$; then there exist some $v \in$ $\mathbb{F}\left(C_{\lambda}\right)$ and $n>0$ such that $d_{\infty}\left({\widehat{D_{\lambda g}}}^{n}(u),{\widehat{D_{\lambda g}}}^{n}(v)\right)<\varepsilon$.

Proof. Since $\left(\mathbb{K}\left(C_{\lambda}\right), \bar{D}_{\lambda}\right)$ is not sensitive, for $\varepsilon>0$ and $\delta>0$, there exist $M \in \mathbb{K}\left(C_{\lambda}\right)$ and $B(M, \delta)$ such that, for all $N \in$ $B(M, \delta)$,

$$
H\left(\bar{D}_{\lambda}^{n}(M), \bar{D}_{\lambda}^{n}(N)\right)<\varepsilon .
$$

Suppose that $u \in e(M)$ (recall that $e(M)=\left\{u \in \mathbb{F}\left(C_{\lambda}\right) \mid\right.$ $\left.[u]_{0} \subseteq M\right\}$ ), and by continuity of $i_{\lambda}$ and (19), we have

$$
\begin{aligned}
H & \left(\bar{D}_{\lambda}^{n}\left([u]_{0}\right), \bar{D}_{\lambda}^{n}(N)\right)<\varepsilon \\
& \Longrightarrow H\left(i_{\lambda} \circ \bar{D}_{\lambda}^{n}\left([u]_{0}\right), i_{\lambda} \circ \bar{D}_{\lambda}^{n}(N)\right)<\varepsilon \\
& \Longrightarrow H\left({\widehat{D} \lambda_{g}}_{n}^{n} \circ i_{\lambda}\left([u]_{0}\right), \widehat{D}_{\lambda g}^{n} \circ i_{\lambda}(N)\right) \\
& =d_{\infty}\left({\widehat{D_{\lambda g}}}_{\lambda}^{n}(u), \widehat{D}_{\lambda g}^{n}(\nu)\right)<\varepsilon .
\end{aligned}
$$

Without loss of generality, assume that $\nu=i_{\lambda}(N) \epsilon$ $\mathbb{F}\left(C_{\lambda}\right)$. This completes the proof.

Remark 17. Theorem 9 together with Theorem 10 shows that $\widehat{f}_{g} M$-chaotic implies $f M$-chaotic, but generally speaking, the converse is not true, which has been discussed in Example 11 and Proposition 16.

\section{Conclusions and Discussions}

In this present investigation, we discuss relations between Martelli chaotic properties of the original and $g$-fuzzified dynamical systems. More specifically, we study stability of the orbits and transitivity and present several examples to illustrate the relations between two dynamical systems. We show that the dynamical properties of the original system and its fuzzy extension mutually inherit some global characteristics. The following main results are obtained.

(a) If $L\left(u_{0}\right)=\mathbb{F}(X)$, then there exists $x_{0} \in\left[u_{0}\right]_{\alpha}^{g}$ such that $L\left(x_{0}\right)=X($ Theorem 10$)$.

(b) The instability of $\operatorname{orb}\left(u, \widehat{f}_{g}\right)$ implies the instability of $\operatorname{orb}(x, f)$, where $u \in \mathbb{F}(X), x \in[u]_{\beta}^{g}$, and $\beta \in[0,1]$ (Theorem 12).

(c) $\widehat{f}_{g} M$-chaotic implies $f M$-chaotic (Theorem 14).

(d) $f M$-chaotic does not imply $\widehat{f}_{g} M$-chaotic (Example 11 and Proposition 16).

It is worth noting that any $g$-fuzzification is connected to a crisp discrete dynamical system in two different ways [19]. One way is to connect two systems via $\alpha$-cut, and another approach is to consider $g$-fuzzified discrete dynamical system as a crisp system that is induced by a certain product map. We develop, in this present paper, the first method. It would be interesting to use the second approach to study the relations between dynamical properties of the original and $g$-fuzzified dynamical systems, and this will be one aspect of our future works.

\section{Conflict of Interests}

The authors declare that there is no conflict of interests regarding the publication of this paper.

\section{Acknowledgments}

This work was partially supported by the National Natural Science Foundation of China (Grant no. 11226268), Scientific and Technological Research Program of Chongqing Municipal Education Commission (Grant no. KJ131219), Postdoctoral Science Foundation of Chongqing (Grant no. Xm201328), and Program for Innovation Team Building at Institutions of Higher Education in Chongqing (Grant no. KJTD201321).

\section{References}

[1] H. Román-Flores, "A note on transitivity in set-valued discrete systems," Chaos, Solitons \& Fractals, vol. 17, no. 1, pp. 99-104, 2003.

[2] H. Román-Flores and Y. Chalco-Cano, "Robinson's chaos in setvalued discrete systems," Chaos, Solitons \& Fractals, vol. 25, no. 1, pp. 33-42, 2005.

[3] A. Peris, "Set-valued discrete chaos," Chaos, Solitons \& Fractals, vol. 26, no. 1, pp. 19-23, 2005.

[4] J. Banks, "Chaos for induced hyperspace maps," Chaos, Solitons \& Fractals, vol. 25, no. 3, pp. 681-685, 2005.

[5] A. Fedeli, "On chaotic set-valued discrete dynamical systems," Chaos, Solitons \& Fractals, vol. 23, no. 4, pp. 1381-1384, 2005.

[6] R. Gu, "Kato's chaos in set-valued discrete systems," Chaos, Solitons \& Fractals, vol. 31, no. 3, pp. 765-771, 2007. 
[7] B. Hou, X. Ma, and G. Liao, "Difference between Devaney chaos associated with two systems," Nonlinear Analysis: Theory, Methods \& Applications, vol. 72, no. 3-4, pp. 1616-1620, 2010.

[8] J. S. Cánovas and J. Kupka, “Topological entropy of fuzzified dynamical systems," Fuzzy Sets and Systems, vol. 165, pp. 37-49, 2011.

[9] J. Kupka, "Some chaotic and mixing properties of Zadeh's extension," in Proceedings of the IFSA World Congress/EUSFLAT Conference, pp. 589-594, Universidade Tecnica de Lisboa, Lisabon, Portugalsko, 2009.

[10] Y. Wang and G. Wei, "Dynamical systems over the space of upper semicontinuous fuzzy sets," Fuzzy Sets and Systems, vol. 209, pp. 89-103, 2012.

[11] H. Román-Flores, Y. Chalco-Cano, G. N. Silva, and J. Kupka, "On turbulent, erratic and other dynamical properties of Zadeh's extensions," Chaos, Solitons \& Fractals, vol. 44, no. 11, pp. 990-994, 2011.

[12] L. Chen, H. Kou, M.-K. Luo, and W. N. Zhang, "Discrete dynamical systems in L-topological spaces," Fuzzy Sets and Systems, vol. 156, no. 1, pp. 25-42, 2005.

[13] R. L. Devaney, An Introduction to Chaotic Dynamical Systems, Addison-Wesley, New York, NY, USA, 2nd edition, 1989.

[14] H. Román-Flores and Y. Chalco-Cano, "Some chaotic properties of Zadeh's extensions," Chaos, Solitons \& Fractals, vol. 35, no. 3, pp. 452-459, 2008.

[15] J. Kupka, "On Devaney chaotic induced fuzzy and set-valued dynamical systems," Fuzzy Sets and Systems, vol. 177, pp. 34-44, 2011.

[16] Y. Y. Lan, Q. G. Li, C. L. Mu, and H. Huang, "Some chaotic properties of discrete fuzzy dynamical systems," Abstract and Applied Analysis, vol. 2012, Article ID 875381, 9 pages, 2012.

[17] L. A. Zadeh, "Fuzzy sets," Information and Computation, vol. 8, pp. 338-353, 1965.

[18] P. Diamond and A. Pokrovskii, "Chaos, entropy and a generalized extension principle," Fuzzy Sets and Systems, vol. 61, no. 3, pp. 277-283, 1994.

[19] J. Kupka, "On fuzzifications of discrete dynamical systems," Information Sciences, vol. 181, no. 13, pp. 2858-2872, 2011.

[20] P. Diamond, "Chaos and fuzzy representations of dynamical systems," in Proceedings of the 2nd International Conference on Fuzzy Logic and Neural Networks, pp. 51-58, FLSI, Iizuka, Japan, July 1992.

[21] W. Huang and X. Ye, "Devaney's chaos or 2-scattering implies Li-Yorke's chaos," Topology and Its Applications, vol. 117, no. 3, pp. 259-272, 2002.

[22] M. Lampart, "Two kinds of chaos and relations between them," Acta Mathematica Universitatis Comenianae, vol. 72, no. 1, pp. 119-127, 2003.

[23] J.-H. Mai, "Devaney's chaos implies existence of $s$-scrambled sets," Proceedings of the American Mathematical Society, vol. 132, no. 9, pp. 2761-2767, 2004.

[24] G. L. Forti, "Various notions of chaos for discrete dynamical systems," Aequationes Mathematicae, vol. 70, no. 1-2, pp. 1-13, 2005.

[25] M. Martelli, M. Dang, and T. Seph, "Defining chaos," Mathematics Magazine, vol. 71, no. 2, pp. 112-122, 1998.

[26] M. Martelli, Introduction to Discrete Dynamical Systems and Chaos, Wiley-Interscience, New York, NY, USA, 1999.

[27] S. Wiggins, Chaotic Transport in Dynamical Systems, Springer, New York, NY, USA, 1991.
[28] P. Diamond and P. E. Klosden, "Characterization of compact subsets of fuzzy sets," Fuzzy Sets and Systems, vol. 29, no. 3, pp. 341-348, 1989.

[29] O. Kaleva, "On the convergence of fuzzy sets," Fuzzy Sets and Systems, vol. 17, no. 1, pp. 53-65, 1985.

[30] H. Liu, E. Shi, and G. Liao, "Sensitivity of set-valued discrete systems," Nonlinear Analysis: Theory, Methods \& Applications, vol. 71, no. 12, pp. 6122-6125, 2009. 


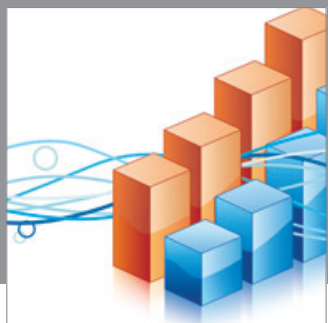

Advances in

Operations Research

mansans

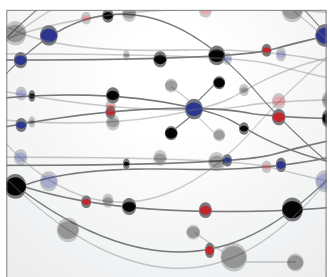

The Scientific World Journal
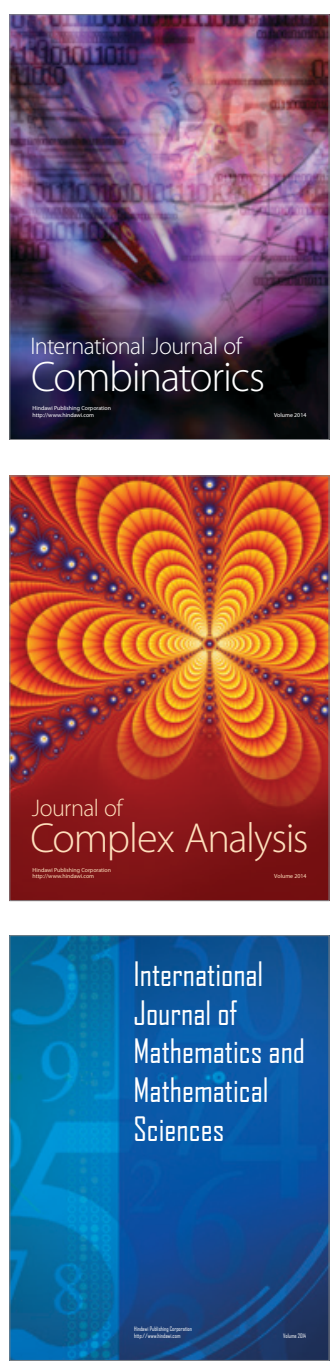
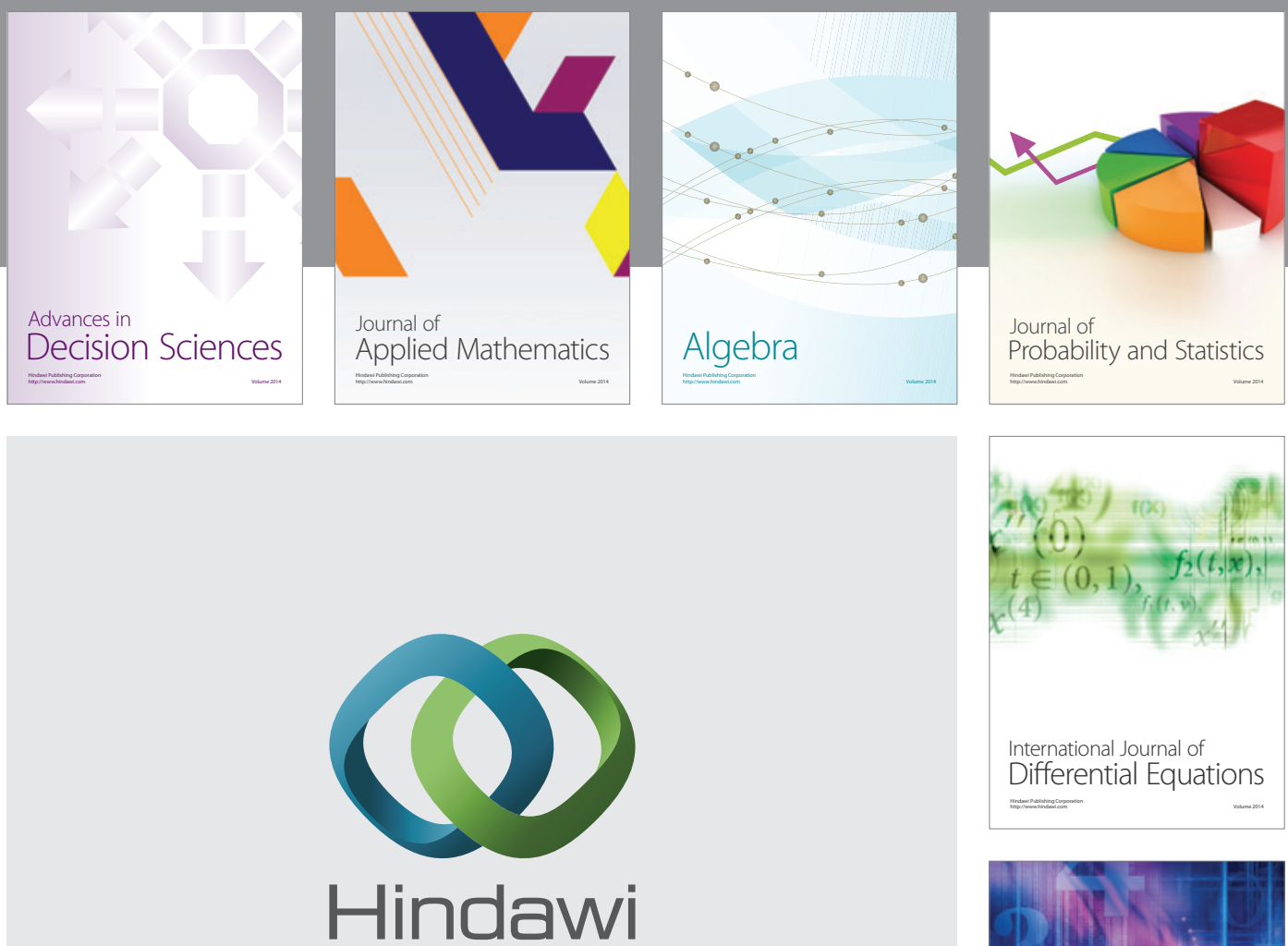

Submit your manuscripts at http://www.hindawi.com
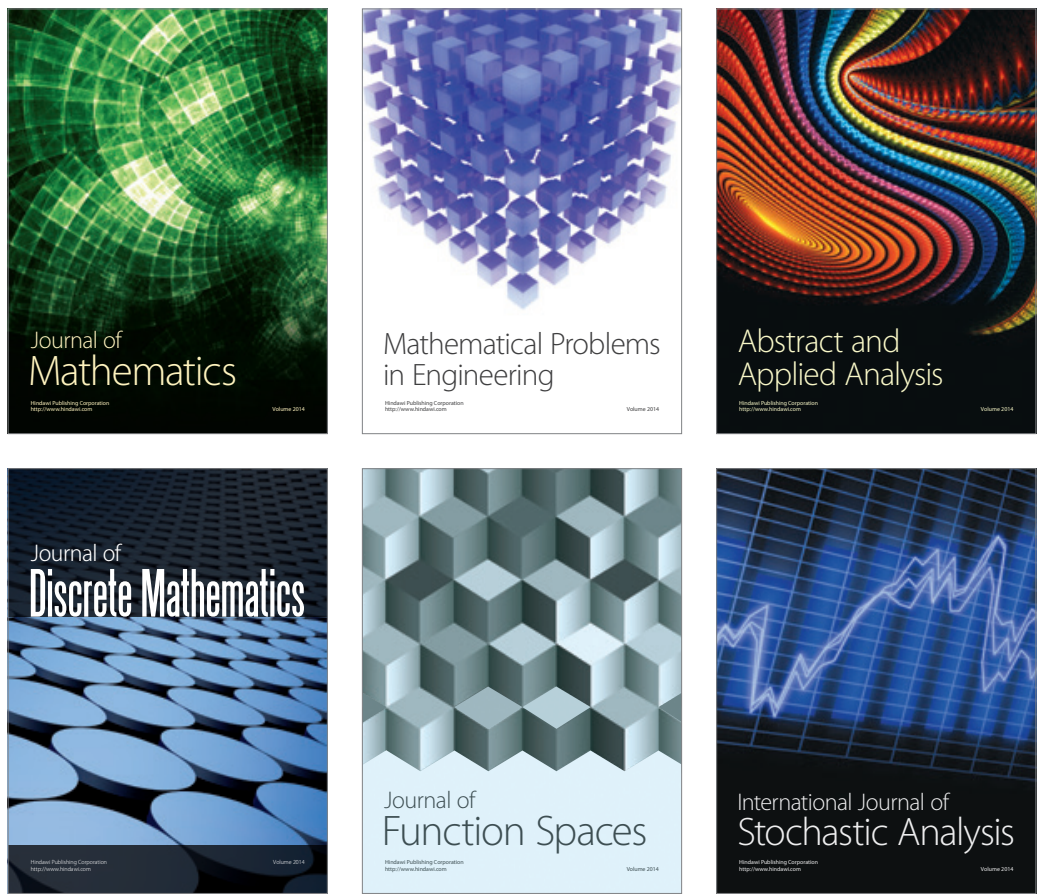

Journal of

Function Spaces

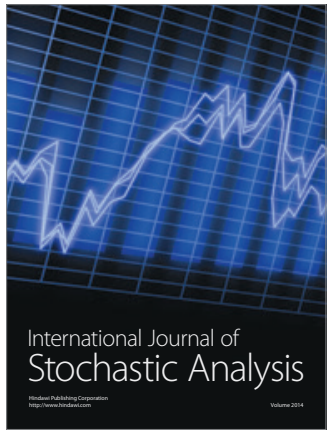

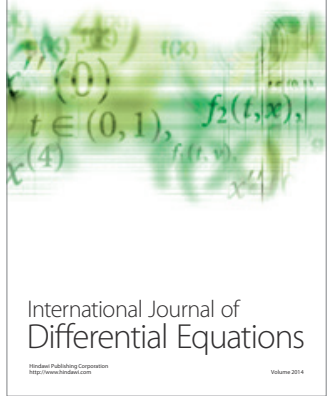
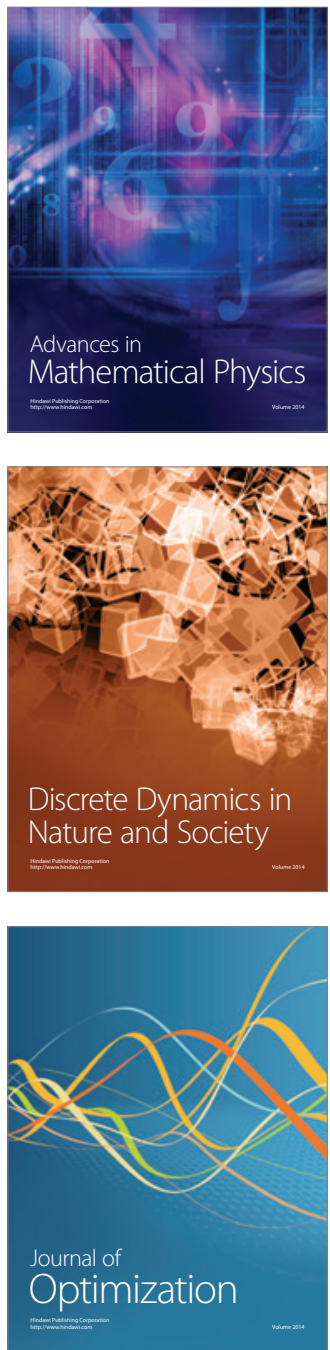